\title{
Triagem neonatal - o desafio de uma cobertura universal e efetiva
}

\author{
Neonatal screening - the challenge of an universal \\ and effective coverage
}

Judy Botler ${ }^{1}$

Luiz Antônio Bastos Camacho ${ }^{1}$

$M$ arly $M$ arques da $\mathrm{Cruz}^{1}$

Pâmela George ${ }^{1}$

\footnotetext{
${ }^{1}$ Escola Nacional deSaúde Pública Sérgio Arouca, Fiocruz. RuaLeopoldo Bulhões 1480/820, Manguinhos. 21041-210 Rio deJaneiro RJ. jbotler@ensp.fiocruz.br
}

Abstract N ewborn screening programs (NSP) aim to detect carriers of several congenital diseases among asymptomatic infants in order to warrant effective intervention. Specimen collection is the first step of a process that should be done in an universal and timely manner. A review of coverageand time of collection wasdonein NSP of several countries. The search was made in various sources, from 1998 to 2008, with "neonatal screening" and "coverage" as key words. The lack of a typical study design did not allow to the rigor required for a systematic review. Data were grouped in macro-regions. Canada had coverage of $71 \%$ in 2006 while the European coverage was of $69 \%$ in 2004, with data of 38 countries. In Asia and Pacific region, there were data of 19 countries. In M iddle East and North Africa, there were data of 4 countries. In Latin A merica, the coverage was 49\% in 2005, with data of 14 countries. In Brazil, coverage was $80 \%$. Twelve reports had information about timeliness. The conclusion is that epidemiological transition has contributed to NSP success. Developed regions had more universal and timelier collection. In Brazil, government initiative increased access to the N SP, but late collections lead to the need of educational actions and participation of professional organizations in developing specific guidelines definition.

Key words Neonatal screening, Coverage, Opportunity, Public Health Program
Resumo Programas de triagem neonatal (PTN) visam detectar portadores de várias doenças congênitas em recém-natos assintomáti cos para uma intervenção efetiva. A coleta do teste é a primeira etapa do processo, devendo ser universal e precoce. Foi feita revisão sobre cobertura e momento de coleta em PTN de diversos países. A busca foi realizada em fontes diversas, de 1998 e2008, com descritores "triagem neonatal" e "cobertura". A falta de desenho de estudo típico impediu o rigor necessário a uma revisão sistemática. Os dados foram agrupados em macrorregiões. 0 Canadá teve cobertura de 71\% em 2006. A Europa teve cobertura de $69 \%$ em 2004, com dados de 38 países. N a Ásia e Pacífico, houve dados de dezenove países. No Oriente M édio e N orte da África, houve dados de quatro países. Na América Latina, a cobertura foi de 49\% em 2005, com dados de catorze países. No Brasil, a cobertura foi de $80 \%$ em 2005. Sobre 0 momento da coleta, houve doze relatos. A transição epidemiológica contribuiu para o êxito dos PT N . Regi ões mais desenvolvidas têm coberturase momento de coleta mais adequados. No Brasil, a iniciativa do governo ampliou o acesso ao teste, mas coletas tardiasindicam a necessi dade de ações educativas e de organizações profissionais na definição de diretrizes específicas.

Palavras-chave Triagem neonatal, Cobertura, Oportunidade, Programas de Saúde Pública 
Introdução

Em saúde pública, triar significa identificar, em uma população assintomática, os indivíduos que estão sob risco de desenvolver determinada doença eque se ben eficiariam de investigação adicional, ação preventiva ou terapêutica imediatas ${ }^{1}$. 0 procedimento de triagem deve ser capaz de alterar a história natural da doença em uma parcela significativa da população el egívele ${ }^{2,3}$.

A triagem neonatal (TN) se baseia na realização de testes laboratoriais nos primeiros dias de vida do recém-nato; esses testes, se feitos no momento e da forma adequados, permitem que 0 início do tratamento ocorra dentro de uma janela de tempo em que é possível evitar sequelas no desenvolvimento da criança4.

As doenças alvo dos programas de triagem neonatal (PTN) ganharam relevância com as mudanças seculares no perfil de morbimortalidaderelacionadas ao desenvolvimento socioeconômico e que caracterizam a chamada "transição demográfica e epidemiológica" 5 . À medida que uma sociedade evolui de um perfil composto por famílias numerosas, com altas taxas de morbidade e mortalidade infantis por causas agudas (desnutrição, doenças infecciosas, etc.), para um padrão de natalidade reduzida, diminuição da mortalidade infantil, aumento na esperança de vida, com um aumento relativo da morbimortalidade por doenças crônicas, a TN passa a assumir importância crescente ${ }^{6}$.

Em outras palavras, quando doenças imunopreveníveis passam a ser equacionadas por medidas preventivas como os programas de vacinação, os sistemas de saúde passam a concentrar sua atenção em doenças menos comuns e mais complexas, porém com efeitos deletérios prolongados sobre o desenvolvimento infantil. O sucesso na prevenção primária de diversas doenças, traduzida pela redução na prevalência destas através de medidas profiláticas (como éo caso de doenças infecciosas), permitiu deslocar esforços para problemas de saúde passíveis de prevenção secundária, ou seja, em que não há como reduzir a prevalência de determinadas doenças, mas o seu diagnóstico e tratamento precoces permitem a redução na incidência das sequelas decorrentes da falta ou atraso nos respectivos tratamentos ${ }^{5}$.

Segundo dados da Organização Mundial de Saúde, estima-se que, em 2005, tenham nascido 136.476 .000 crianças no mundo ${ }^{7}$, das quais $2 \%$ somente no Brasili. Para Horovitz et al. ${ }^{8}$, se, no Brasil, na década de oitenta, as doenças congêni- tas ocupavam o quinto lugar como causa de mortalidadeem crianças menores deum ano, em 2000, estas passaram a assumir o segundo lugar, refletindo a mel hora da qualidade dos cuidados perinatais e aproximando o Brasil do perfil de mortalidade neonatal dos países mais desenvolvidos.

O marco introdutório dos PTN, na década de sessenta, foi o desenvolvimento da metodologia para dosagem de fenilalanina em amostras de sangue seco colhidas em cartões de papel filtro, permitindo o diagnóstico da fenilcetonúria $(\mathrm{PKU})^{4}$.

Esta doença metabólica, apesar da baixa prevalência (média mundial em torno de 1:15.000 nascidos vivos), apresenta graves efeitos sobre 0 desenvolvimento do sistema nervoso quando não tratada adequadamente e no momento oportuno. 0 acúmulo de fenilalanina tem efeitos tóxicos sobre o sistema nervoso em formação, levando ao retardo mental, que pode ser evitado com uma dieta precoceespecífica com baixosteores deste aminoácido ${ }^{10}$.

A utilização de cartões de papel filtro como veículo para coleta de amostras de sangue permitiu a ampliação do acesso ao teste a grandes populações, facilitando o transporte do material coletado em localidades remotas via correio postal até um laboratório central.

$N$ a década de setenta, Dussault (Canadá) desenvolveu metodologia para dosagem de tiroxina (T4) e tireotrofina (TSH) no mesmo tipo de amostra ${ }^{11}$. 0 hipotireoidismo congênito - doença caracterizada pela deficiência na produção do hormônio tireoidiano - tem prevalência média mundial em torno de 1:3.500 nascidos vivos e também podelevar ao retardo mental senão tratado da forma e no momento adequados ${ }^{12}$. A Academia Americana de Pediatria (AAP) e a Associação Americana de Tireóide afirmam que 0 tratamento instituído até quinze dias de vida é capaz de garantir o desenvolvimento neurológico normal mesmo nos casos mais graves ${ }^{13}$.

N as quatro décadas de sua existência, a TN se revelou um programa de saúde pública fundamental, capaz de evitar a morte e o retardo mental $^{12,14}$. Ela evoluiu de um teste laboratorial para uma única doença - PKU - para um conjunto de ações de controle bem mais abrangentes e complexos ${ }^{15}$. Ao longo desta trajetória, houve grandes avanços tecnológicos, com a introdução de novas técnicas laboratoriais, que ampliaram de forma exponencial as possibilidades diagnósticas.

Com os avanços nessa área, surgiu a necessidade de definição de critérios para seleção de doenças para que estes esforços se traduzissem em 
programas de saúde de fato efetivos. Em 1967, a Organização Mundial de Saúde criou uma forçatarefa que definiu oscritérios deWilson eJ ungner para que uma doen ça fosse can didata a fazer parte de PTN no nível da saúde pública. Quarenta anos após a sua publicação, estes critérios ainda são considerados "padrão ouro" na seleção de doenças, a saber: a doença triada deve ser considerada como um importante problema de saúde pública; deve existir tratamento disponível para os casos detectados; deve haver infraestrutura para confirmação diagnóstica etratamento específicos; deve haver um período de tempo, antes do início dos sintomas, em que a intervenção melhore 0 desfecho; deve haver um teste de triagem adequado e confiável; a história natural da doença deve ser bem conhecida (incluindo o período entre a doença latente e declarada); deve haver um protocolo quedeixeclaro quem deveser tratado como paciente; o programa deve ser custo-efetivo; e a busca de casos deve ser um processo contínuo ${ }^{16}$.

Segundo documento da Secretaria deV igilância em Saúde(SVS) do M inistério da Saúdebrasileiro, uma condição representa um problema de saúde pública quando tem algumas características, dentreas quais: magnitude(doenças com elevada frequência, que afetam grandes populações, com altastaxas deincidência, prevalência, mortalidade e anos potenciais de vida perdidos); transcendência (características que conferem relevância especial à doença - como severidade - potencial de letalidade, hospitalização e sequelas; relevância social - valor imputado pela sociedade, como medo, repulsa ou indignação; e relevância econômica - capacidade de causar prejuízos, como restrições comerciais, redução na força de trabalho, absenteísmo escolar e laboral, custos assistenciais e previdenciários); e vulnerabilidade (medida pela disponibilidade de instrumentos específicos de prevenção e controle da doença, propiciando a atuação efetiva dos serviços desaúde sobre os indivíduos e coletividades) ${ }^{17}$.

As doenças mais comumente diagnosticadas pelos PTN apresentam uma magnitude relativamente pequena, porém de elevada transcendência (em todos os seus aspectos) e vulnerabilidade. Este grupo de doenças apresenta frequências bastante variáveis, porém com potencial mórbido, incapacitante e causador de sequelas de tal ordem que tornam sua severidade, relevância social eeconômica indiscutíveis.

No Brasil, o Ministério da Saúde criou, em 2001, o Programa Nacional de Triagem Neonatal (PNTN) ${ }^{18}$ com meta de cobertura universal em território brasileiro ea garantia de realização de todas as etapas que vão da coleta até o tratamento e acompanhamento dos casos detectados. Para tanto, foi previsto o custeio de todas as etapas pelo Sistema Ú nico de Saúde.

A heterogeneidade demográfica, econômica, política, cultural, logística e de diversas outras naturezas de um país como o Brasil faz com que todas as etapas deste amplo processo representem um grande desafio. A coleta dos testes éapenas o primeiro passo nesta sequência e é afetada por toda a sorte de dificuldades.

Para efeito de seleção do painel de doenças, 0 PNTN foi organizado em três fases de implantação, para as quais cada estado foi credenciado com base na cobertura e infraestrutura preexistentes: fasel - hipotireoidismo congênito ePKU; fase II - hipotireoidismo congênito, PKU e hemoglobinopatias; fase III - hipotireoidismo congênito, PKU, hemoglobinopatias efibrose cística.

Os PTN, se estruturados de forma adequada, comportam-se como ferramentas que permitem uma intervenção efetiva sobre os indivíduos afetados, permitindo que os mesmos possam ter um desenvolvimento pleno. 0 impacto social se reflete na redução dos custos de manutenção de indivíduos incapacitados e das perdas laborativas ${ }^{19}$.

Este tipo de programa compreende estruturas bastante complexas que envolvem uma ampla rede de unidades de coletas, laboratório especializado, um sistema de busca ativa, e estrutura para confirmação diagnóstica, tratamento multidisciplinar e acompanhamento no longo prazo ${ }^{20}$. Estas atividades são desempenhadas por diversas categorias profissionais de saúde, em locais distintos, e costumam estar subordinadas a diferentes gestores. Ainda assim, devem estar bem articuladas de forma a garantir que o programa alcance pleno êxito em seus objetivos ${ }^{20}$.

0 caráter assintomático dessas doenças na sua fase inicial ea inexistência de um grupo considerado de alto risco tornam essencial a busca pelo acesso universal ao teste para todos os recémnatos vivos de uma determinada localidade.

Entre as diversas dimensões de acesso desenvolvidas por Pechansky e Thomas ${ }^{21}$, os PTN se defrontam com três: disponibilidade (relação entre o volume etipo de serviços e recursos existentes e o volumeetipo denecessidades dos usuários); acessibilidade (relação entre a localização do serviço e a localização dos usuários, levandose em conta os recursos necessários para que 0 usuário entre em contato com os serviços disponíveis); adequação (relação entre como os recursos oferecidos são organizados para admitir os 
usuários e a habilidade dos usuários de se ajustarem à forma como estes são oferecidos).

A realização da coleta, primeira de uma sequência de etapas fundamentais do processo de triagem, passa a ser crítica, tendo-seem vista a grande variedade de problemas que podem incidir sobre a mesma. Além do acesso universal, o momento adequado da coleta (oportunidade) também é fundamental para o bom desempenho do sistema de TN. Como o teste inclui o diagnóstico de doenças metabólicas, sua coleta deve ser feita com tempo suficiente para que ocorra acúmulo do metabólito analisado no sangue (tempo mínimo de 48 horas de alimentação), mas sem ultrapassar o tempo máximo em que é possível se evitar as complicações decorrentes do início tardio do tratamento. A AAP estabelece que as coletas devam ser realizadas entre 0 terceiro e quinto dias de vida, jamais ultrapassando o sétimo ${ }^{22}$.

0 sucesso desta etapa depende de envolvimento de autoridades sanitárias, campanhas educativas incluindo profissionais de saúde e população, investimentos financeiros, e uma ampla rede de estruturas organizacionais com características próprias ${ }^{20}$.

Dada a importância da cobertura da coleta dos testes para o bom desempenho de todo 0 processo da TN, neste trabalho, pretende-se fazer uma busca mais abrangente possível sobre a situação da cobertura dos PTN em diversas regiões no mundo, assim como questões relativas ao momento da coleta, buscando traçar um paralelo entre a situação do programa brasileiro e seus equivalentes em outros países. Os achados foram organizados de forma a, ao final, setentar uma visão mais ampla do "estado da arte" sobre a questão da abrangência e grau de oportunidade das coletas, com um olhar especial sobre a situação do programa brasileiro. As etapas subsequentes à triagem não farão parte do escopo desta revisão.

\section{Material e métodos}

Os estudos que abordam as questões relativas à cobertura e o momento das coletas geralmente se apresentam na forma narrativa, sem um padrão metodológico que permita sistematizá-los de acordo com determinado desenho de pesquisa. Elaborados em formatos bastante diversos, não permitem o rigor necessário a uma revisão sistemática.

Foram utilizados, como fontes de informação, ferramentas de busca na Internet (Bibliote- ca Cochrane, Pubmed, M edcarib, Wholis, Lilacs eSciELO), artigos em periódicos, jornais especializados, livros, documentos oficiais de alguns países e anais de eventos nacionais e internacionais relacionados à TN. Os descritores utilizados foram os termos triagem neonatal e cobertura, assim como seus congêneres em inglês (neonatal screening e coverage) e espanhol (tami saje neonatal e cobertura).

A estratégia de busca incluiu trabalhos que mencionavam dados de cobertura; questões relativas ao acesso ao teste; momento da coleta ou qualidade da amostra colhida; novas propostas para se alcançar a cobertura efetiva; e dificuldades para implantação de PTN ; no período entre 1998 e 2008, priorizando-se sempre o dado mais recente de cada localidade.

Foram excluídos da seleção: avanços tecnológicos na área de TN e confirmação diagnóstica; discussões sobre inclusão de novas doenças nos painéis de TN ; questões éticas; aspectos genéticos, clínicos eevolutivos relativos às doenças triadas; tratamentos; trabalhos sobre doenças diferentes daquelas que fazem parte do painel do programa brasileiro; TN auditiva, de distúrbios oculares e de outras malformações congênitas.

Para melhor compreensão e visualização, os dados foram compilados, conforme apresentados nos resultados, em figuras e tabelas segundo as regiões correspondentes no mundo: Figura 1 - Europa, Figura 2 - Ásia e região do Pacífico; Figura 3 - América Latina.

Como a maioria das figuras foi elaborada a partir de dados obtidos em trabalhos publicados que abordavam de forma global as respectivas regiões, a autoria e ano de publicação foram mencionados como referência no título das tabelas, exceto para dados isolados que, por serem mais recentes, mereceram uma citação a parte.

No caso da América do Norte, N orte da África e Oriente M édio, as informações foram descritas na forma narrativa. 0 Brasil recebeu tratamento diferenciado e na apresentação de seus resultados, como será visto a seguir, é possível observar as coberturas parciais relativas aos diversos estados da federação segundo as respectivas fases de implantação em que foram credenciados para o PNTN .

Cabe mencionar que a questão do momento da coleta foi abordada à parte em virtude da diversi dade nas formas de apresentação destes dados. Estes foram descritos na maneira como são citados nos respectivos textos. 


\section{Resultados}

Os resultados obtidos na literatura foram expostos deforma agrupada por continentee, aquelesrelativos ao Brasil, apresentados deforma destacada, para que tornasse possível a comparação entre a situação do programa brasileiro e os seus semelhantes em outras regiões do mundo.

Para efeito desta publicação, a América do Norte ${ }^{23}$ foi representada pelos Estados Unidos e - Canadá. Em ambos os países analisados, não há políticas nacionais para a TN. Por isso, a evolução dos PTN se deu de forma bastante heterogênea entre os respectivos estados, províncias e territórios.

Os estados norte-americanos têm leis estaduais que torna a TN obrigatória e uma responsabilidade desaúde pública. Naquele país, o sistema desaúdeécusteado de diferentesmaneiras, sendo aproximadamente cinquenta por cento com recursos privados (seguros de saúde). 0 governo financia al gumas atividades para os mais pobres e idosos e tem uma participação variável nos diversos estados quanto ao custeio dos PTN ${ }^{23}$.

A AAP recomenda que a coleta seja feita no momento da alta hospitalar ${ }^{22}$. A alta hospitalar precoce passa a representar um problema, já que induz às coletas em prazo inferior a vintee quatro horas de vida. Por isso, nove estados norte-americanos tornaram uma segunda coleta obrigatória para todos os recém-natos, a ser realizada quando estes al cançam a idade de uma ou duas semanas de vida. Não há informação sobre a cobertura global dos Estados Unidos para a primeira coleta. A proximadamente $25 \%$ dos recém-natos são submetidos a uma segunda coleta.

Aliado a este protocolo, os esforços do governo norte-americano e de organizações médicas no sentido de promover o compartilhamento eintegração entre ossistemas dedados desaúde neonatal e os de TN levam a supor que a cobertura deste programa seja universal em todos os estados da federação e que o momento da coleta seja inferior ao preconizado pela maioria dos programas ao redor do mundo (entre o terceiro eo sétimo dia de vida); porém, estefato é corrigido pela segunda coleta23.

No Canadá, apenas uma província (Saskatchewan) tem lei que obriga a TN para a PKU e o hipotireoidismo congênito. As demais províncias e territórios "confiam nos padrões da boa prática médica como encorajamento para a TN adequada". O governo não tem qual quer participação nos PTN, nem existequalquer estratégia nacional, padrões ou diretrizes queorientem os seus desempe- nhos. Nenhuma organização profissional de saúde canadense assumiu qualquer posição pública relativaàTN nosúltimosanos. Em março de2006, aproximadamente $71 \%$ dos recém-natos canadenses tiveram acesso aos testes, com distribuição bastante heterogênea pelas províncias²3.

0 governo financia praticamente todo o sistema de saúde. Todos os cidadãos e imigrantes têm direito ao seguro desaúde público, quelimita 0 acesso para alguns hospitais e médicos. A participação privada no financiamento da saúde fica em torno de $30 \%{ }^{23}$.

Diante deste quadro, cada estado, província ou território, norte-americano ou canadense, realiza TN para um número bastantevariável de doenças. Os serviços de busca ativa, tratamento e acompanhamento em ambos os países são patrocinados pelo setor público, privado ou uma combinação de ambos. A parentemente, a maior preocupação em ambos os países é com a incorporação de tecnologia para inclusão de novas doenças. A discussão sobre estratégias para criação de programas bem articulados, que visem todas as etapas que vão até o tratamento e acompanhamento dos casos, e os problemas gerados neste tipo desistema, parece ocorrer em segundo plano.

No que se refere à Europa ${ }^{24}$, o desenvolvimento da TN naEuropa tem sido lento eheterogêneo - mais rápido nos países ocidentais e mais Iento no Leste Europeu. O Conselho Europeu consiste de quarenta e cinco países-membros, com uma população de recém-natos estimada em 8,8 milhões, em 2004. Estima-se que a cobertura global da TN em toda a Europa, naquele ano, tenha sido em torno de $69 \%^{24}$. Este dado pode estar subestimado, visto que, no levantamento realizado por Loeber ${ }^{24}$, não há informações de alguns países como Albânia, Armênia, Azerbaijão, Georgia, Hungria, M acedônia, Turquia e M oldávia.

A informação parcial sobre o Reino Unido obtida por Loeber foi complementada neste trabalho com os dados fornecidos pelo relatório oficial do U K N ewborn ScreeningP ProgrammeCentre; entretanto, estedado se refereao ano de 2007. Todos os países que compõem o Reino Unido (Inglaterra, Escócia, Irlanda do Norte e País de Gales) alcançaram a cobertura universal ${ }^{25}$.

$\mathrm{Na}$ Figura 1, são observadas as coberturas parciais dos programas existentes na Europa. 0 grupo de colunas situado na parte superior do gráfico, que vão da Romênia à Estônia, se refere aos países do LesteEuropeu. 0 grupo subsequente inclui países da Europa O cidental. 
Cobertura global estimada Bósnia-H erzegovina

Romênia

Sérvia

U crânia

Bielo-Rússia

Polônia

Eslováquia

Bulgária

Croácia

Lituânia

Letônia

Rússia

Eslovênia

República Tcheca

Estônia

Chipre

Bélgica

Portugal

Finlândia

$M$ alta

Inglaterra*

Escócia

I rlanda do Norte*

Luxemburgo

País de Gales

Grécia

Suíça

Suécia

Alemanha

Noruega

Holanda

Islândia

Dinamarca

Irlanda

Áustria

Itália

França

Espanha
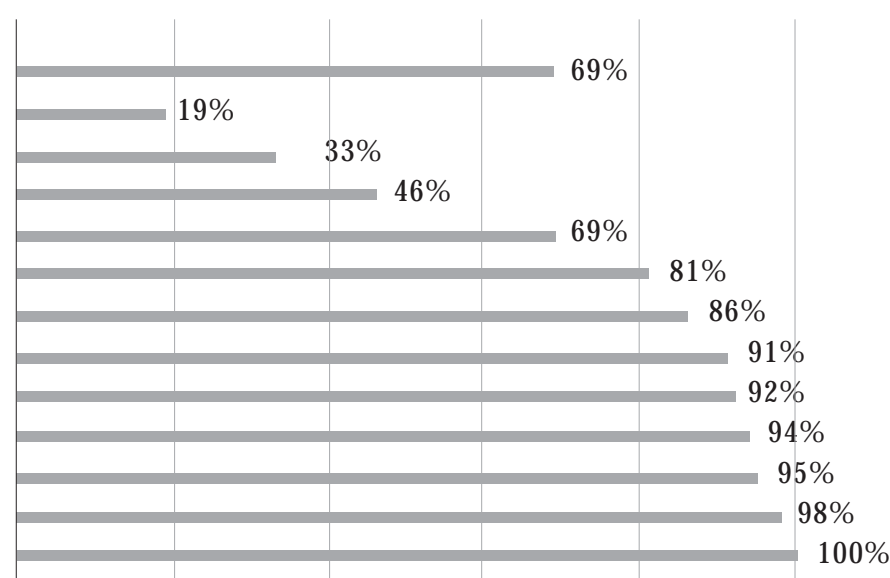

$100 \%$
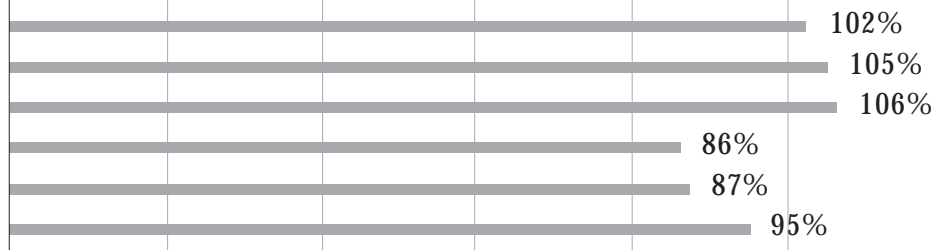

$95 \%$

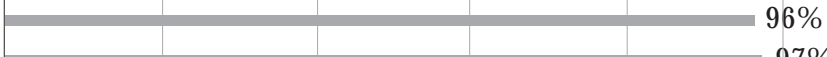

\begin{tabular}{|r|r|r|r|}
\hline & $97 \%$ \\
\hline & $98 \%$
\end{tabular}
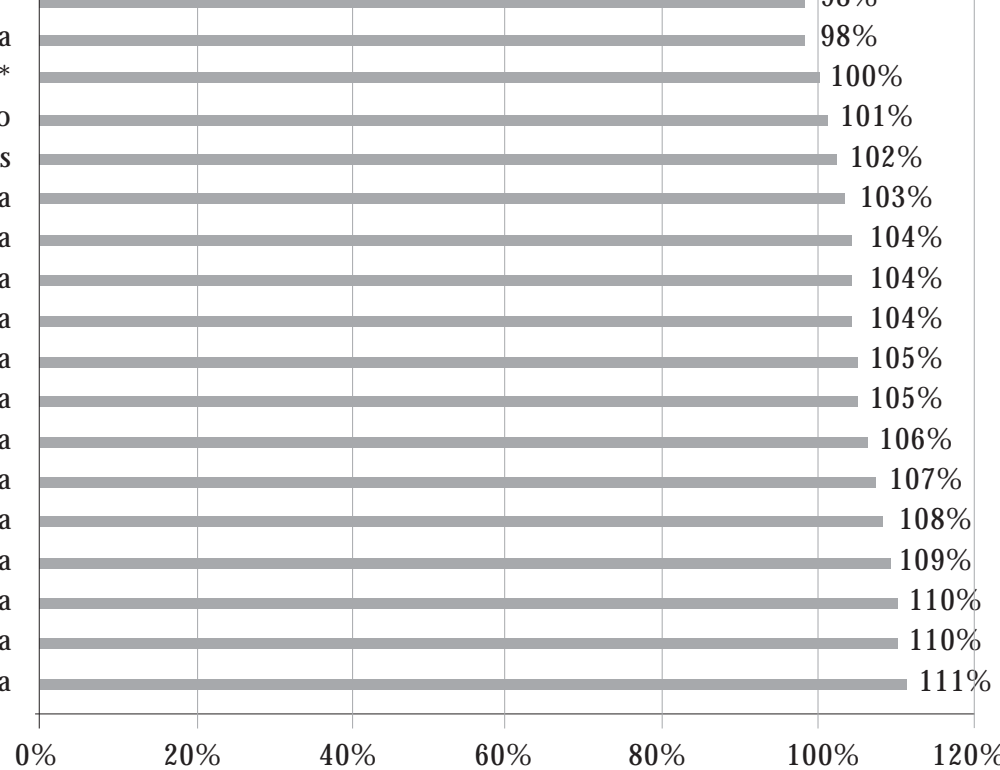

* Reino U nido (Inglaterra el rlanda do N orte) foram obtidos de um relatório bianual publicado pelo governo britânico em 2008 (relativo ao biênio 2006-1007) ${ }^{25}$.

** Países como Albânia; Armênia; Azerbaijão; Geórgia; H ungria; M acedônia; eTurquia não foram citados por não haver informações disponíveis sobre os respectivos PTN .

*** Os recém-natos de Liechtenstein eM ontenegro são triados na Suíça e Sérvia, respectivamente.

**** M oldávia eU crânia não têm programas nacionais.

Figura 1. Cobertura da triagem neonatal na Europa em 2004 (modificado de Loeber ${ }^{24}$ ). 
Se uma cobertura igual ou superior a $95 \%$ dos nascidos vivos for considerada satisfatória, é possível notar que, com exceção de Chipre e Bélgica, todos os países da Europa Ocidental já alcançaram a cobertura universal. Alguns países apresentam, no levantamento de Loeber, coberturas superiores a $100 \%$, o que pode ser explicado por imprecisões nas bases de dados de nascidos vivos e/ou dos PTN ${ }^{24}$. Em particular, a cobertura de $104 \%$ na Suíça pode ser parcialmente explicada pelo fato daquele país incorporar a TN dos recém-natos de Lichstenstein.

N os países ocidentais, a preocupação se concentra em identificar nichos populacionais em que há risco de omissão na coleta (como crianças de origem africana ou migrantes) ${ }^{26}$ e atividades que tornem a coleta mais precoce, como um sistema de cruzamento entre as bases de dados de nascimentos e as dos PTN ${ }^{27}$.

A incorporação de novas tecnologias e introdução de novas doenças nos respectivos programas éfeita de maneira bastante cautel osa. No Rejno Unido, o PTN éuma iniciativa administrada e custeada pelo governo e se encontra bem articulado para garantir todas as etapas envolvidas até 0 tratamento. Desta forma, a decisão pela inclusão denovas doenças leva em conta os critérios deWilson e Jungner ${ }^{16,28}$ e estudos sobre a relação custoefetividade para cada uma das doenças ${ }^{29}$.

Nos países do Leste Europeu, o grau de desenvolvimento dos programas é bastante diversificado e as respectivas coberturas podem variar de 19\% (Bósnia-H erzegovina) a 106\% (Estônia). Nestes países, questões de natureza política, logística, cultural e econômica contribuem para a heterogeneidade da implementação dos PTN . Ainda assim, seis dos quinze países alcançam a cobertura universal - Lituânia, Letônia, Rússia, Eslovênia, República Tcheca e Estônia. Países como Bósnia-H erzegovina, Romênia, Sérvia, Ucrânia, Bielo-Rússia, Polônia, Eslováquia, Bulgária, Croácia e Lituânia ainda apresentam problemas de acesso ao teste traduzidos em coberturas insuficientes.

Segundo dado da UNICEF, das 134 milhões de crianças que nasceram em 2007 no mundo, aproximadamente metade nasceu na Ásia e países do Oceano Pacífico ${ }^{30}$. Destas, $80 \%$ nasceram em apenas cinco países - China, Indonésia, Bangladesh, Índia e Paquistão. Em países desta região em quea taxa de mortalidade infantil é menor do que 10/1.000 nascidos-vivos, os PTN têm alcançado coberturas superiores a $90 \%$. Entre os demais países, com mortalidade infantil superior, a Tailândia (taxa de mortalidade infantil de
13/1.000 nascidos-vivos) foi o único a alcançar uma boa cobertura de TN ( 97\%).

Nos países em que a cobertura tem sido insuficiente, a implementação dos PTN vem se deparando com diversos obstáculos, que incluem economias pobres, educação em saúde insuficiente, falta de apoio governamental, alta hospitalar precoceeelevado número de partos domiciliares. Na maioria dos países em desenvolvimento na região do Pacífico, aproximadamente $80 \%$ dos nascimentos ocorre fora do ambiente hospita$\operatorname{lar}^{30}$. Somam-se a estas questões, diferenças de idioma e cultura, além de grandes variações geográficas (países muito extensos, um número expressivo de pequenas ilhas e muitas regiões montanhosas) e governos instáveis.

Segundo Padilla ${ }^{30}$, para que os PTN da região alcancem toda a população, há necessidadedepriorização dos mesmos pelos governos; financiamento governamental (total ou parcial); educação e aceitação por parte do público; maior envolvimento dos profissionais de saúde e participação do governo na institucionalização dos sistemas deTN. 0 quese observaéque existem diferenças substanciais nas legislações dos países com relação à TN. Se, por um lado, as leis chinesas são permissivas com relação ao desenvolvimento gradual do seu sistema de TN, por outro, nas Filipinas, as leis são mais assertivas sobre os profissionais que atendem o recém-nato na hora do parto quanto à obrigatoriedade da coleta dos testes.

Com todas essas dificuldades, os PTN vêm se desenvolvendo desde a década de sessenta de forma bastante heterogênea. Os programas mais evoluídos são observados nas regiões economicamente mais desenvolvidas. A Nova Zelândia foi o primeiro país da região a ter um programa nacional de TN . O Japão foi o primeiro a desenvolver um programa de controle de qualidade laboratorial para este tipo de programa. A Austrália foi um dos primeiros países no mundo a utilizar a tecnologia da espectrometria de massa.

$\mathrm{Na}$ Figura 2, são expostas as coberturas estimadas para a maioria dos países da região. Fica patente a heterogeneidade nas coberturas, ficando as coberturas plenas restritas aos países com intensa atividadeeconômica. N essecontexto, cabe destacar a situação da China que, embora apresente coberturas parciais entre 80 e $90 \%$ nas regiões de Shanghai, Beijing, Tianjin e Guagnzhou, tem cobertura global de apenas a $25 \%$, deixando evidentea grandedificuldade de acesso aos serviços de saúde ${ }^{30}$.

Os países do O riente M édio e N orte da Áfri$\mathrm{ca}^{31}$ são classificados como "em desenvolvimen- 
to". A maioria destes pertence à Liga Árabe e 0 idioma predominanteéo árabe. Com exceção da Argélia, territórios palestinos e Israel, todos são membros do Eastern M editerranean Regional O ffice (EM RO) da Organização M undial da Saúde. A Argélia pertence ao Regional Office for Africa e os territórios palestinos e I srael, ao Regional Office for Europe.

O grupo de países ligado ao EM RO inclui: M arrocos, Tunísia, Líbia, Egito, Sudão, Somália, Djibuti, Jordânia, Líbano, Síria, Arábia Saudita, Bahrain, Qatar, Emirados Árabes, Kuwait, Iraque, Iêmen, Omã, Irã, Afeganistão e Paquistão. Quinze por cento das crianças que morrem atéo primeiro ano de vida no mundo nascem em sete países desta região - o que equivale 1,5 milhões deóbitos a cada ano. A proximadamente metade destas morre no período neonatal por asfixia, prematuridade ou quadros infecciosos graves. Nos demais países em que tem havido declínio na mortalidade infantil, as autoridades de saúde vêm adquirindo maior conscientização materializada em políticas direcionadas para as doenças genéticas e malformações congênitas. N estes países, 20 a $50 \%$ dos casamentos são consanguíneOS, as famílias são numerosas, a idade dos pais costuma ser elevada. Estes fatos favorecem à elevada incidência de distúrbios metabólicos gené ticos, hematológicos, além de outros defeitos congênitos.

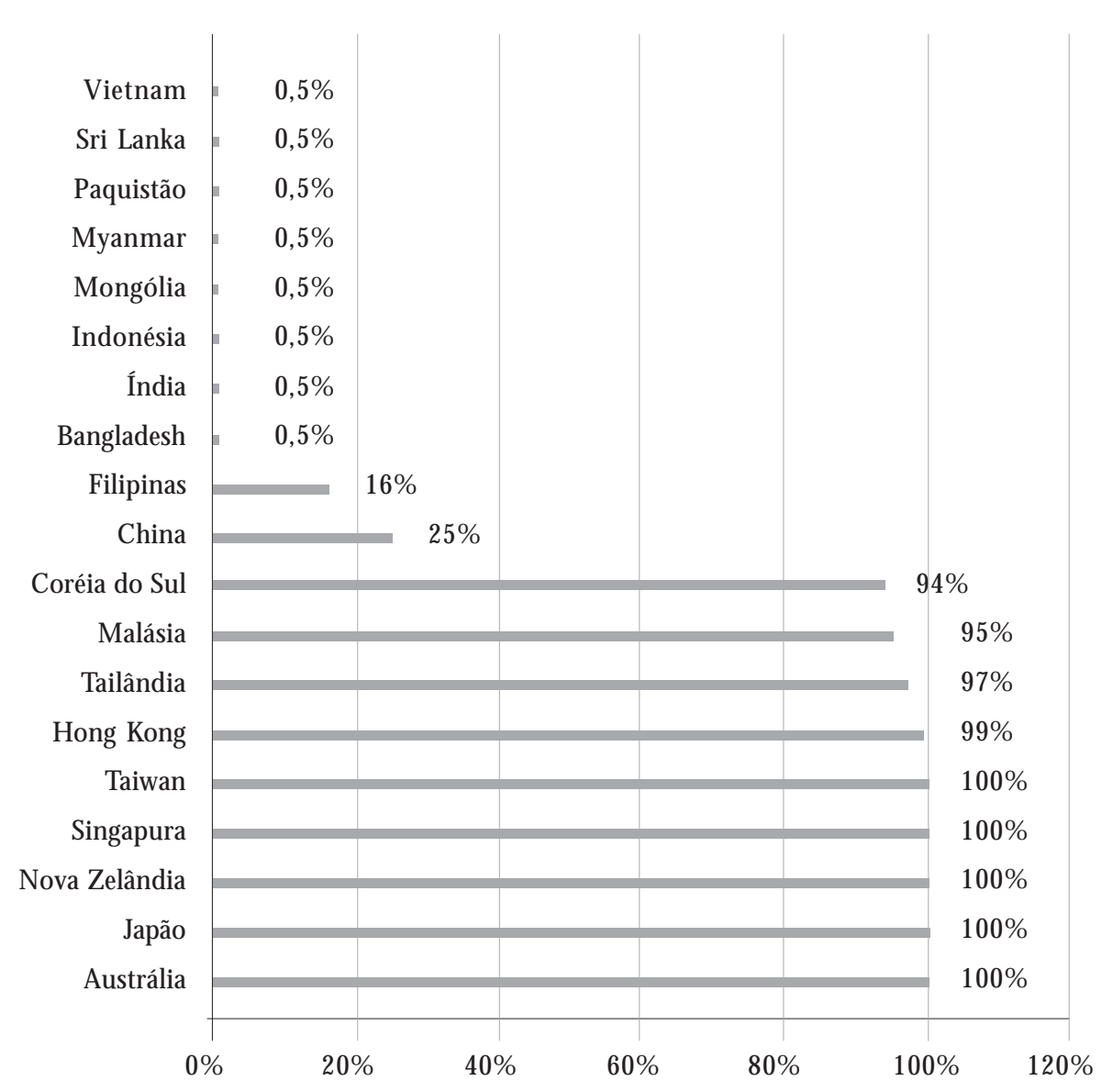

* N ão há informações sobre a triagem neonatal no Cambodja, Coréia do N orte, Laose N epal.

** Palau recentementefez um acordo para realização dos testes detriagem neonatal em uma parceria com as Filipinas.

Figura 2. Cobertura dos PTN na Ásia e região do Pacífico em 2006 - modificado de Padillaº. 
A "Declaração de Marrakech", de 2006, definiu a TN para doenças congênitas como uma prioridade para os programas de saúde pública desta região. Países como Bahrein, Egito, Irãä Jordânia, Kuwait e Arábia Saudita iniciaram estudos-piloto para TN de al gumas doenças como hemogl obinopatias, deficiência de glicose-6-fosfato dehidrogenase, hipotireoidismo congênito, hiperplasia adrenal congênita, fibrosecística, PKU e outras doenças metabólicas.

Apenas cinco países desta região têm PTN nacionais: Egito, Omã, Qatar, Arábia Saudita e Emirados Árabes. 0 programa egípcio teve cobertura estimada em torno de 75\% em 2003. Na A rábia Saudita, em 2006, estima-se que a cobertura tenha alcançado $25 \%{ }^{31}$. Os Emirados Árabes alcançaram uma cobertura de $65 \%$, em 2000 , com distribuição heterogênea ${ }^{33}$. Não há informações sobre a situação da TN na Argélia e Israel. Entretanto, entre os territórios palestinos, há registro de um programa na Faixa de Gaza para triagem dePKU, com cobertura estimada em 35\% em $2000^{34}$.

A América Latina éuma região composta por vinte países: M éxico, Costa Rica, El Salvador, Guatemala, Honduras, Nicarágua, Panamá, Cuba, República Dominicana, Haiti, Argentina, Bolívia, Brasil, Chile, Colômbia, Equador, Paraguai, Peru, Uruguai e Venezuela. Anualmente, nascem aproximadamente 11,2 milhões de crianças na América Latina e, em 2005, 49\% destas foram submetidas à TN. Estes países se caracterizam por enormediversidade geográfica, demográfica, étnica, econômica e de sistemas sociaise de saúde. Com exceção do Brasil e H aiti, o espanhol é o idioma dominante. Estas diversidades encontram paralelo nos principais indicadores sociais e de saúde dos países da região ${ }^{35}$.

O Brasil compreende $43 \%$ da extensão geográfica e $34 \%$ da população de toda a América Latina. Por esta razão e para efeito da revisão realizada, foi dado maior destaque para o programa brasileiro, que acabou recebendo tratamento diferenciado em outra seção deste trabal ho.

A TN na América Latina teveinício na década desetenta, a partir deiniciativasisoladas, no Mé xico (Velázquez) eBrasil (Schmidt), com a detecção da PKU. A expansão da TN como programa nacional para os demais países encontrou muitas dificuldades de ordem financeira, política, cultural elogística35.

Para Borrajo ${ }^{35}$, os países da América Latina podem ser agrupados em seis categorias, conforme o grau de implementação e cobertura dos respectivos PTN : Grupo I (Cuba, Costa Rica,
Chile eU ruguai) - países com os programas mais organizados. As coberturas estão em torno de $100 \%$ etodas as etapas da TN até o tratamento e acompanhamento estão a cargo dos respectivos governos; Grupo II (Brasil, M éxico eArgentina) - países com sistemas de saúde bastante complexos. Por muitos anos, o setor privado foi o maior responsável pela execução efinanciamento das atividades de TN, alcançando coberturas entre 60 e 80\% em 2005. 0 Brasil se destacou em relação aos demais após a criação do seu PNTN (em 2001), quando o governo passou a assumir o controleda TN no nível nacional; Grupo III (Colômbia, Paraguai e Venezuela) - a implementação de programas no nível nacional é bem mais recente (começando em 1999), com coberturas inferiores às observadas nos grupos I ell; Grupo IV (Nicarágua e Peru) - os PTN só começaram a se organizar nos últimos dois anos e suas coberturas estão entre 4\% e6\%; Grupo V (Guatemala, República Dominicana, Bolívia, Panamá eEquador) - não existequal quer iniciativa no nível nacional. As atividades de TN são mínimas, realizadas primordialmente pelo setor privado, com coberturas inferiores a 1\%; Grupo VI (El Salvador, Honduras eH aiti) - as atividades de TN são praticamenteinexistentes.

$\mathrm{Na}$ Figura 3, são apresentadas as coberturas dos PTN dos países dos grupos I a IV. Os demais não foram incluídos por suas atividades serem pouco expressivas ou inexistentes.

No Brasil, a TN foi iniciada a partir de 1976 com ações isoladas, sem qualquer orientação governamental. De acordo com dados do M inisté rio da Saúde, em 2000, a cobertura no país era em torno de $55 \%$ dos nascidos vivos, distribuídos de forma desigual. 0 governo, por sua vez, financiava apenas os testes de triagem e não havia qualquer comprometimento com o tratamento e acompanhamento dos casos afetados ${ }^{36}$.

A criação do PNTN pelo Ministério da Saúde, em 2001, definiu como objetivos a cobertura universal e a garantia de acesso ao tratamento e acompanhamento qualificados. Todas estas etapas passaram a ser financiadas pelo governo e foi definida uma hierarquia de responsabilidades ecompetências nas esferas federal, estadual e municipal.

Os estados foram credenciados em uma de três fases de acordo com as doenças contempladas, tendo como fatores condicionantes a cobertura einfraestrutura preexistentes. Assim, foram credenciados treze estados para a fase I, que incluía a PKU e o hipotireoidismo congênito; nove estados para a fase II, que, além das doenças já 
citadas na fase I, incluía as hemoglobinopatias; e três estados na fase III, que também incluía a fibrose cística.

A criação de serviços de referência em triagem neonatal estaduais, articulados a amplas redes de coleta e serviços diagnósticos e assistenciais, elevou a cobertura global estimada para aproximadamente $80 \%$ em $2005^{37}$, porém deforma bastante heterogênea (Figura 4).

$\mathrm{Na}$ Figura 4, procurou-se apresentar o dado de cobertura mais recente para cada estado. A maior parte dos dados foi publicada por Carva$\mathrm{Iho}^{37}$. As exceções couberam aos estados do $M$ ato Grosso do Sul ${ }^{38}$, Rio Grande do Sul ${ }^{39}$, M inas Gerai $s^{40}$, Santa Catarina ${ }^{41}$, Sergipe ${ }^{42}$ e Rio de Janeiro (dados informados diretamente pela Secretaria de Estado de Saúde e Defesa Civil do Rio de Janeiro referentes ao ano de 2006). Os estados de Alagoas, Distrito Federal e Pernambuco não forneceram os respectivos resultados.

As estimativas de cobertura de Carvalho et al. ${ }^{37}$ tiveram como numerador os totais de testes realizados por cada estado em 2005 e como denominador os totais de nascidos vivos de cada estado em 2004 (dado disponível no Sistema de Nascidos Vivos do DATASUS na época). Talvez isso explique algumas imprecisões nos dados apresentados, como éo caso do Paraná, queapresenta cobertura de $112 \%$.

As maiores coberturas se encontram nos estados credenciados para a fase III e o inverso se dá com aqueles credenciados para a fase I. Cabe res-

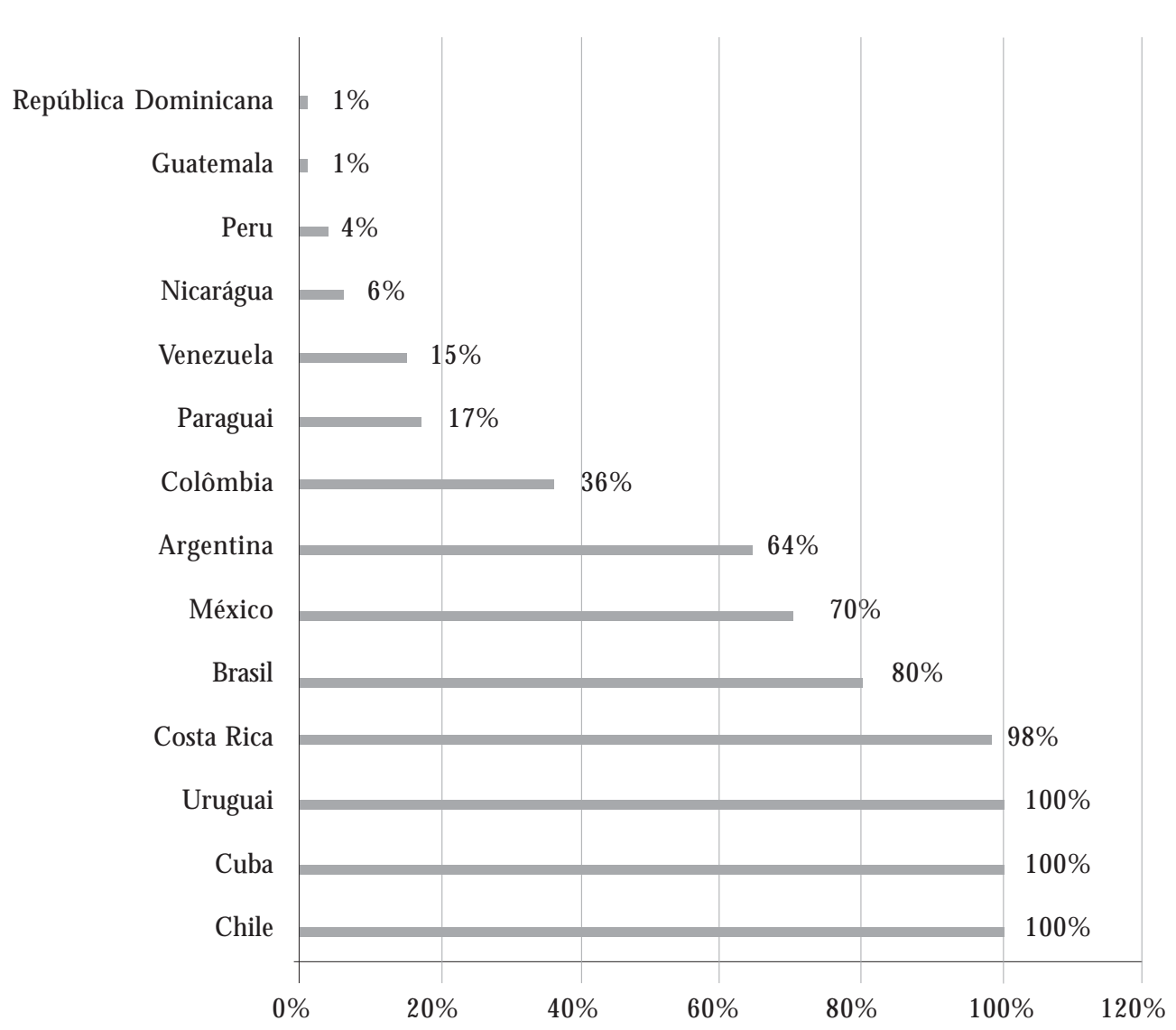

* Bolívia, Panamá, Equador, El Salvador, Honduras e H aiti não foram incluídos porque as atividades de triagem neonatal nestes países são mínimas ou inexistem.

** Em 2005, estima-se que a cobertura global da triagem neonatal na América Latina tenha sido em torno de 49\%.

Figura 3. Cobertura dos PTN na América Latina, em 2005 (modificado de Borrajo ${ }^{35}$ ). 


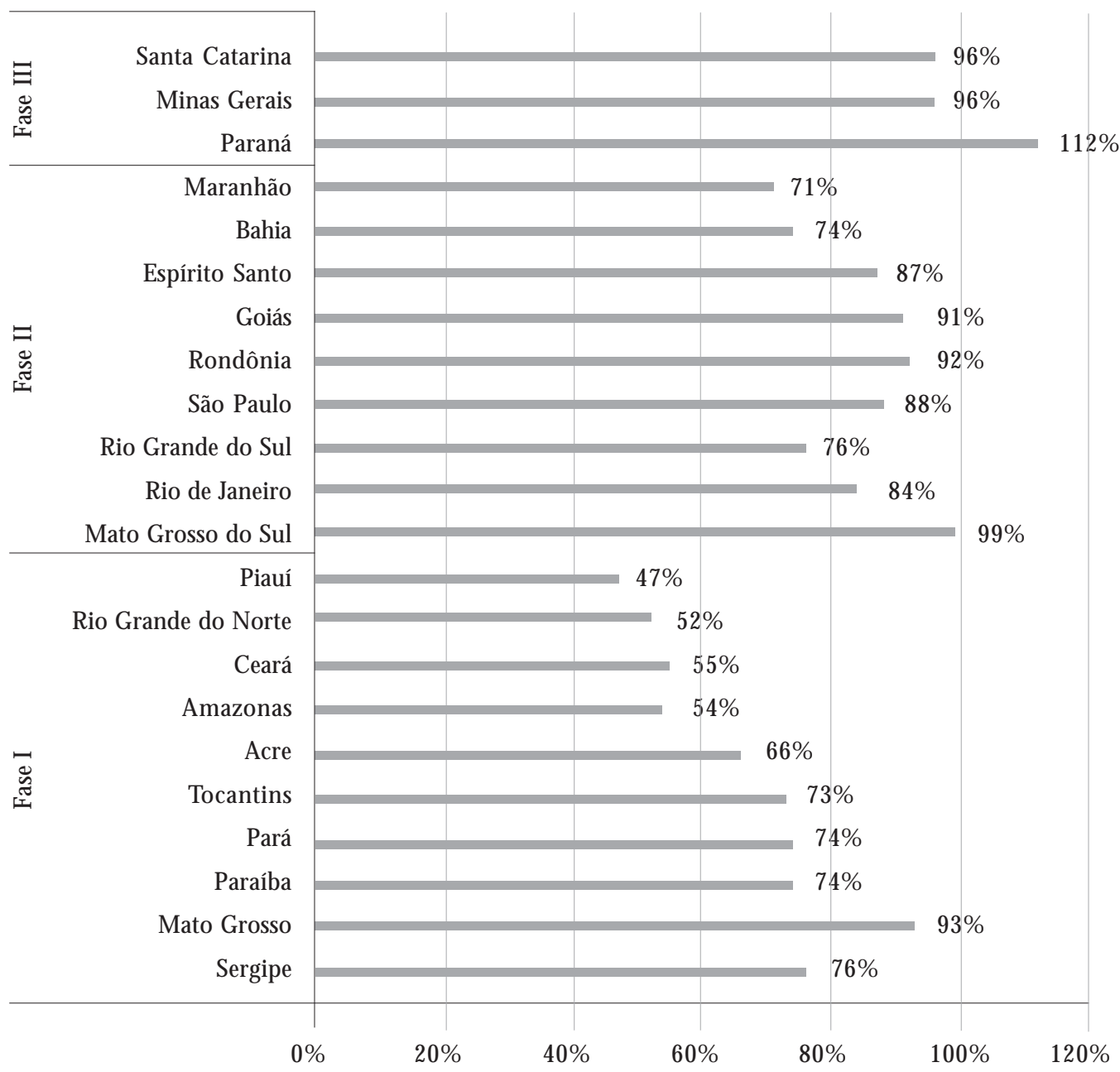

* O cálculo apresentado por Carvalho representa uma estimativa, já que os totais de testes de cada estado se referem ao ano de 2005 e o denominador de nascidos vivos se refere ao ano de 2004.

** Os dados relativos aos estados do M ato Grosso do Sul ${ }^{38}$, Rio Grande do Sul ${ }^{39}$, M inas Gerai ${ }^{40}$, Santa Catarina ${ }^{41}$ e Sergi pe ${ }^{42}$ foram obtidos de publicações isoladas mais recentes que o levantamento feito por Carvalho.

*** Os estados de Alagoas, Distrito Federal (Fase I) e Pernambuco (Fase II) não forneceram dados sobre sua cobertura.

**** A cobertura do estado do Rio de Janeiro se refere ao ano de 2006 e os dados de triagem neonatal enascidos vivos foram obtidos diretamente da Secretaria de Estado de Saúde e D efesa Civil daquele estado e Coordenação Estadual do PNTN.

Figura 4. Cobertura estimada dos PTN nos diversos estados brasileiros, organizados pela fase de implantação em que foram credenciados para o PNTN em 2005 (modificado de Carvalho ${ }^{37 *}$ ).

saltar que os estados do Mato Grosso (fase I) e M ato Grosso do Sul (fasell) apresentam coberturas muito discrepantes em relação aos demais estados que se encontram nas mesmas fases. Como ambos são estados fronteiriços com países vizinhos, como a Bolívia (que não tem um programa nacional organizado), é de se supor que migrações possam interferir nesta estimativa. 
De uma forma geral, há uma expressiva heterogeneidade na distribuição das coberturas, que provavel mente tem relação com a situação dos indicadores de desenvolvimento econômico, social, político, cultural e de saúde dos respectivos estados. Os estados em melhor situação são os que têm melhor cobertura e o inverso se dá nos estados onde estes indicadores têm pior desempenho.

\section{Oportunidade da coleta}

Para efeito de comparação, será considerada como referência a diretriz estabelecida pelaAAP ${ }^{22}$, que estabel ece que as coletas não devem superar o sétimo dia de vida do recém-nato.

Os dados dos trabalhos obtidos se encontram na Tabela 1 e são apresentados de forma bastante diversificada. Por isso, os mesmos serão apenas comentados, tendo em vista a impossibilidade de compará-los.

Dos doze trabalhos em que se observa esta informação, nove se referem aos estados brasileiros. Entre os três restantes, há o de Coppinger (Reino Unido) ${ }^{25}$, de 2007, mostrando que, além da ótima cobertura (98\%), mais de $90 \%$ das coletas se deu entre cinco e oito dias de vida. Nos Emirados Árabes, em 2003 ${ }^{33}$, apesar da baixa cobertura ( $65 \%$ ), $98 \%$ das coletas se deram antes dos dez dias de vida. Na Faixa de Gaza34, além da cobertura baixa ( $35 \%)$, a maioria das coletas se deu em momento inoportuno ( $61 \%$ das coletas entre onze e dezessete dias).
No Brasil, apenas nove estados publicaram seus cálculos de tempo para coleta do teste: Bahia $^{43}$, Sergipe ${ }^{42}$, Paraíba ${ }^{44}$, Cearáa ${ }^{45}$ M inas Gerais $^{40}$, Espírito Santo ${ }^{46}$, Santa Catarina ${ }^{41}$, Paraná ${ }^{47}$ e Rio Grande do Sul ${ }^{39}$. Apesar das disparidades na forma de apresentação, é possível observar que, nos estados onde a cobertura se encontra mais longe da meta (Figura 4), as coletas são feitas mais tardiamente (Ceará, Paraíba, Bahia, Sergipe, Rio Grande do Sul eEspírito Santo). 0 oposto se observa nos estados com meIhor cobertura (M inas Gerais, Santa Catarina e Paraná).

Talvez não por acaso, os estados em que a cobertura universal é acompanhada de menor tempo de coleta são aquel es que se encontram na fase III do PNTN. Isso talvez revele uma melhor organização no acesso ao teste e, possivelmente, na estruturação dos respectivos programas. Os estados com menor cobertura etempos mais longos para a coleta se encontram nas fases I e II, e estes dados sugerem a necessidade de maior investimento em medidas locais que facilitem 0 acesso ao teste para que as coberturas se tornem de fato universais e as coletas oportunas.

\section{Discussão ecomentáriosfinais}

Conforme o exposto, a literatura mostra que, nas suas quatro décadas de existência, a TN se disseminou pelo mundo de forma bastante desigual. As transições epidemiológica e demográfica se

Tabela 1. Programas que apresentaram estimativa de intervalo de tempo entre nascimento e a coleta dos testes para triagem neonatal.

\begin{tabular}{|c|c|c|c|c|}
\hline Autor & Ano & País/Estado & Localidade & $\begin{array}{l}\text { Tempo entre } \\
\text { nascimento e coleta }\end{array}$ \\
\hline Coppinger ${ }^{25}$ & 2007 & Reino Unido & Global & $\begin{array}{l}\text { Exceto } M \text { anchester, todas as regiões tiveram } \\
\geq 90 \% \text { das coletas entre } 5 \text { e } 8 \text { dias }\end{array}$ \\
\hline Al-Hosani et al. ${ }^{33}$ & 2003 & Emirados Árabes & Global & $98 \%$ das coletas $\leq 10$ dias \\
\hline Shahla et al. ${ }^{34}$ & 2004 & Territórios Palestinos & Faixa de Gaza & $61 \%$ das coletas entre 11 e 17 dias \\
\hline Almeida et al. ${ }^{43}$ & 2003 & Bahia/Brasil & Global & $85 \%$ das coletas $\geq 7$ dias \\
\hline Ramalho et al. ${ }^{42}$ & 2007 & Sergipe/Brasil & Global & M édia de $10 \pm 9$ dias \\
\hline Ramos et al. ${ }^{44}$ & 2003 & Paraíba/Brasil & Campina Grande & M édia de $18 \pm 12$ dias \\
\hline Ribeiro et al. ${ }^{45}$ & 2005 & Ceará/Brasil & Global & Maioria $>60$ dias \\
\hline Chagas $^{40}$ & 2003 & M inas Gerais/Brasil & Global & M ediana de 7 dias \\
\hline Hegner et al. ${ }^{46}$ & 2005 & Espírito Santo/Brasil & Global & M ediana de 15 dias \\
\hline N ascimento ${ }^{41}$ & 2002 & Santa Catarina/Brasil & Global & $49 \%$ até 7 dias \\
\hline Carvalho et al. ${ }^{47}$ & 2005 & Paraná/Brasil & M aringá & $92 \%$ das coletas $\leq 5$ dias \\
\hline Pinto et al. ${ }^{39}$ & 2005 & Rio Grande do Sul/Brasil & Global & M édia de 11 dias \\
\hline
\end{tabular}


mostraram fatores determinantes para 0 avanço dos PTN . Nas localidades onde o padrão de famílias numerosas com altas taxas de mortalidade infantil foi substituído por famílias menores com indicadores de saúde neonatal mel hores, pode-se observar um aumento expressivo na cobertura desses programas. A relação entre os dois fenômenos é possivelmente indireta, já que ambos dependem denível socioeconômico.

O impacto das deficiências em PTN não é fácil de ser avaliado, considerando que as doenças alvo não são de notificação compulsória em muitos países. A frequência de sequelas e complicações destas doenças pode ser considerada como parâmetro para avaliação do programa como um todo, incluindo a triagem e acompanhamento das crianças, cuja eficácia já é bem conhecida.

No LesteEuropeu, al gumas regiões da Ásia e América Latina estas diferenças são bem acentuadas. No Brasil, o mesmo efeito pode ser notado através das diferenças entre os programas do Norte e Nordeste do país quando comparados aos estados do Sul e Sudeste.

0 envolvimento dos governos locais e das sociedades profissionais tem se mostrado crítico não apenas com relação à cobertura, mas também com relação ao grau de oportunidade das coletas.

É curioso observar a situação da América do Norte, berço dos PTN, onde a participação governamental praticamenteinexiste. Emboraexista grande preocupação com incorporação de novas tecnologias, tanto nos Estados Unidos quanto no Canadá, ainda é possível se observar uma cobertura insuficiente no Canadá.

Nos Estados Unidos, a AAP cumpre um papel orientador com diretrizes que salientam a importância da coleta dos testes ainda no ambiente hospitalar, levando a supor que, dessa forma, a cobertura seja de fato universal e a coleta dos testes, oportuna.

$\mathrm{Na}$ Europa Ocidental, onde os programas têm intensa participação do governo, as coberturas alcançam a totalidade dos nascidos vivos, as coletas são oportunas e todos os demais processos, bem articulados, garantindo o pleno êxito em todas as etapas dos respectivos programas. Nestes países, há um grande cuidado na seleção das doenças triadas, já que o financiamento dos programas é integralmente coberto pelos respectivos governos $s^{28,29}$.

No LesteEuropeu, há grande heterogeneidade em todos os aspectos da TN. Ainda existem países como Bósnia-H erzegovina, Romênia, Sér- via eU crânia em que a cobertura dos respectivos programas se mostra muito aquém do ideal.

$\mathrm{Na}$ Ásia e países do Pacífico, são observados os grandes contrastes de cobertura e organização dos PTN. De um lado, encontramos países como Austrália, N ovaZelândia eJapão, onde não apenas a cobertura é universal e as coletas oportunas, como ainda há a preocupação com a identificação de grupos de risco para omissão na coleta (como é 0 caso da população aborígene) ${ }^{48}$. De outro lado, vemos países como Filipinas e China enfrentando toda sorte de problemas para a implementação dos respectivos programas. A China, apesar do intenso crescimento econômico, demonstra claramente os problemas inerentesaum país degran de extensão territorial egrande iniquidade no acesso aos serviços de saúde. N os grandes centros econômicos, a cobertura é plena, porém a cobertura global não passa dos vinte e cinco por cento da população neonatal.

O N orte da África e Oriente M édio, aparentemente, pelos dados disponíveis, encontram-seno início das discussões sobreimplementação da TN .

A América Latina, por sua vez, apresenta o mesmo padrão de heterogeneidade de cobertura visto na Ásia, enfrentando problemas bastante semel hantes para a implementação de seus programas.

No Brasil, a criação do PNTN ${ }^{18}$ foi fundamental para 0 avanço da cobertura. M esmo assim, o acesso ao teste ainda émuito heterogêneo, com cobertura menor em estados com menor envolvimento governamental, e maior nos estados onde os respectivos órgãos administrativos têm uma atuação mais participativa. Quadro semelhante se dá com relação ao grau de oportunidade das coletas. Em que pese à participação de instituições que não compõem a rede pública de serviços de saúde, a sua articulação com a rede pública torna-se fundamental para o pleno êxito dos respectivos programas. Em alguns estados, este tem sido um fator complicador para o bom desempenho destes sistemas, enquanto em outros, este desafio já parece ter sido ultrapassado.

A participação das sociedades profissionais no programa brasileiro ficou restrita à Sociedade Brasileira de Endocrinologia e M etabologia, que criou um grupo de trabalho para discutir o problema ${ }^{49}$. A Sociedade Brasileira de Pediatria selimitou apenas a incluir a legislação criada pelo M inistério da Saúde no seu website, sem emitir qualquer orientação específica aos pediatras.

A pesar da existência do PNTN, ainda persistem questões geográficas, políticas econômicas, sociais, culturais e educacionais ( da população e 
dos profissionais de saúde) que podem contribuir para as grandes dificuldades de acesso ao teste ${ }^{50}$.

Para finalizar, é importante salientar que a coleta do testeéa primeira deuma sériedeetapas que fazem parte do processo de TN. Portanto, vale a pena ressaltar o artigo publicado por Wang ${ }^{51}$, mostrando a experiência da província de Shandong (China), onde a cobertura é de setenta por cento, as coletas são feitas por parteiras no terceiro dia de vida; entretanto, as amostras de sangue podem levar até quatro semanas para chegar ao laboratório. Assim fica demonstrado que o processo de $\mathrm{TN}$ requer uma estrutura ampla e complexa, em que a coleta é apenas o primeiro passo. Sem uma articulação adequada com as demais etapas, os programas se tornam ineficazes em sua proposta de tratamento precoce e prevenção de sequelas para o desenvolvimento infantil.

\section{Colaboradores}

J Botler participou da coleta e análise dos dados, formatação dos gráficos e tabelas e elaboração do texto final. LAB Camacho participou da análise dos dados e revisão final. M M Cruz participou da revisão final e P George participou da elaboração dos gráficos e da revisão final. 


\section{Referências}

1. Wald N. The definition of screening. J Med Screening 2001; 8:1.

2. Holland W. Screening for disease - considerations for policy. Euro Observer 2006; 8(3):1-4.

3. Peckham C, Desateux C. Issues underlying the evaluation of screening programmes. Br M ed Bull 1998; 54(4):167-178.

4. Therrell B. U.S. Newborn screening policy dilemmas for the twenty-first century. $\mathrm{M}$ ol Gen $\mathrm{M}$ etab 2001; 74(1-2):64-74.

5. Omran AR. The epidemiological transition: a theory of the epidemiology of population change: 1971 M ilbank Mem Fund Q 2005; 83(4):731-757.

6. Pollitt R. Introducing new screens: Why are all doing different things? J Inherit M etab Dis 2007; 30(4):423-429.

7. World Health Organization. [site da Internet]. [acessado 2007 ago 3]. Disponível em: http:// www.who.int/topics.

8. Brasil. M inistério da Saúde. [site da Internet]. [acessado 2007 ago 3]. Disponível em: http:// www.datasus.gov.br

9. Horovitz D, Mattos RA, Llerena-J r JC. Atenção aos defeitos congênitos no Brasil: panorama atual. Cad Saude Publica 2005; 21(4):1055-1064.

10. Scriver C, Kaufman S, Eisensmith RC, Woo SLC. The $H$ yperphenylalaninemias. In: Scriver $C$, Beaudet AL, Sly WS, Valle D, editors. The M etabolic and Molecular Basis of Inherited Disease. New York: McGraw-Hill; 1995. p. 1015-1076.

11. Botler J. Análise crítica da implantação de um programa de rastreamento para hipotireoidismo congênito no Estado do Rio de Janeiro [dissertação]. Rio de Janeiro (RJ): Pontifícia Universidade Católica; 1992.

12. Botler J. Repercussões neurológicas do hipotireoidismo congênito. Arq Bras de Endocrinol M etab 1996; 40(4):264-270

13. American Academy of Pediatrics, Rose SR; Section on Endocrinology and Committee on Genetics, American Thyroid Association, Brown RS; Public Health Committee, Lawson Wilkins Pediatric Endocrine Society, Foley T, Kaplowitz PB, Kaye Cl, Sundararajan S, Varma SK . Update of Newborn Screening and Therapy for Congenital Hypothyroidism. Pediatrics 2006; 117(6):2290-2303.

14. Rovet J, Daneman D. Congenital hypothyroidism: a review of current diagnostic and treatment practices in relation to neuropsychologic outcome. Pae diatric Drugs 2003; 5(3):141-149.

15. Pass K, Lane PA, Fernhoff PM, Hinton CF, Panny SR, Parks JS, Pelias MZ, Rhead WJ, Ross SI, Wethers DL, Elsas LJ 2nd. US N ewborn screening system guidelines II: Follow-up of children, diagnosis, management and evaluation statement of the Council of Regional Networks for Genetics Services (CORN). J Pediatr 2000; 137(4 Suppl):S1-S6.

16. Andermann A, Blancquaert I, Beauchamp S, Déry V. Revisiting Wilson and Jungner in the genomic age: a review of screening criteria over the past 40 years. Public Health Classics - Bull WHO 2008; 86(4):317-319.
17. Brasil. M inistério da Saúde. Secretaria de Vigilância em Saúde. Departamento de Vigilância Epidemiológica. Guia de Vigilância Epidemiológica. Brasília: M inistério da Saúde; 2005.

18. Brasil. M inistério da Saúde. Portaria GM/no 822, de 6 de junho de 2001. Cria o Programa Nacional de Triagem Neonatal (PNTN). Diário Oficial da União 2001; 7 jun.

19. Ward LS, Maciel RMB, Magalhães RF, Kunii IS, Kurazawa GK, Matsumura LK, Vieira JGH. Estimativa da relação custo-benefício de um programa de detecção precoce do hipotiroidismo congênito. Rev Ass M ed Bras 1988; 34(3)106-110.

20. Webster D. Quality performance of newborn screening systems: strategies for improvement. J Inherit M etab Dis 2007; 30(4):576-584.

21. Pechansky R, Thomas JW. The concept of access definition and relationship to consumer satisfaction. M edical Care 1981; 19(2):127-140.

22. American Academy of Pediatrics. Issues in newborn screening. Pediatrics 1992; 89(2):345-349.

23. Therrell BL, Adams J. Newborn screening in North America. J Inherit M etab Dis 2007; 30(4):447-465.

24. Loeber J. Neonatal screening in Europe: the situation in 2004. J Inherit M etab Dis 2007; 30(4):430-438.

25. Coppinger $C$, Cavanagh $C$. Data Collection \& Performance Analysis Report. In: Antenatal and Newborn Screening Programmes. UK: N ewborn Screening Programme Centre; 2008.

26. Simpson N, Randall R, Lenton S, Walker S. Audit of neonatal screening programme for phenylketonuria and congenital hypothyroidism. Arch Dis Child Fetal Neonatal Ed. 1997; 77(3):228-234.

27. Ades A, Walker J, Jones R, Smith I. Coverage of neonatal screening: failure of coverage or failure of information system. Arch Dis Child 2001; 84(6):476479.

28. Lord J, Thomason MJ, Littlejohns P, Chalmers RA, Bain MD, Addison GM, Wilcox AH, Seymour CA. Secondary analysis of economic data: a review of cost-benefit studies of neonatal screening for phenylketonuria. J Epidemiol Community $\mathrm{H}$ ealth 1999; 53(3):179-186.

29. Thomason MJ, Lord J, Bain MD, Chalmers RA, Littlejohns P, Addison GM, Wilcox AH, Seymour CA. A systematic review of evidence for the appropriateness of neonatal screening programmes for inborn errors of metabolism. J Public Health $\mathrm{Med}$ 1998; 20(3):331-343.

30. Padilla CD, Therrell BL. Newborn screening in the Asia Pacific region. J Inherit M etab Dis 2007; 30(4):490-506.

31. Saadallah A, Rashed MS. Newborn screening: experiences in the Middle East and North Africa. J Inherit M etab Dis 2007; 30(4)482-489.

32. Ordookhani A, Mirmiran P, Hajipour R, Hedayati $M$, Azizi F. Screening for congenital hypothyroidism in the Islamic Republic of Iran: strategies, obstacles and future perspectives. East $M$ editerr $\mathrm{H}$ ealth J 2002; 8(4\&5):632. 
33. Al-Hosani H, Salah H, Saade D, Osman H, Al-Zahid J. United Arab Emirates National Newborn Screening Programme: an evaluation 1998-2000. Rev Santé M editerranée Orientale 2003; 9(3):324-332.

34. Shahla ANKA, Abed Y, Shahla NKA. Screening programme for phenylketonuria in the Gaza Strip: evaluation and recommendations. J Trop Pediatr 2004; 50(2):101-105.

35. Borrajo G. Newborn screening in Latin America at the beginning of the 21st century. J Inherit Metab Dis 2007; 30(4):466-481.

36. Carvalho TM, Santos HP, Santos ICGO, Vargas PR, Pedrosa J. Newborn screening: a national public health programme in Brazil. J Inherit M etab Dis 2007; 30(4):615.

37. Carvalho TM. Triagem Neonatal no Brasil. Rev M éd de M inas Gerais 2005; 15(2 Suppl.1):20-22.

38. Botelho CAO, Botelho MA. Programa de triagem neonatal de M ato Grosso do Sul. Rev M éd de M inas Gerais 2005; 15(2 Suppl.1):107.

39. Pinto AB, Wajner M, Goldbeck AS, Vargas PR. Programa de Triagem Neonatal para Fenilcetonúria e Hipotireoidismo Congênito no Rio Grande do Sul. Rev. M éd. de M inas Gerais 2005; 15(2-Suppl.1):61.

40. Chagas AJ. Rastreamento neonatal em M inas Gerais. In: M edeiros N eto $\mathrm{G}$, organizador. Hipotireoidismo congênito no Brasil - como era, como estamos, para onde vamos. São Paulo: Aché; 2003. p. 37-43.

41. Nascimento ML. Hipotireoidismo congênito em Santa Catarina. In: Medeiros Neto G, organizador Hipotireoidismo congênito no Brasil - como era, como estamos, para onde vamos. São Paulo: Aché; 2003. p. 53-61.

42. Ramalho ARO, Ramalho RJR, Oliveira CRP, Santos EG, Oliveira MCP, Aguiar-Oliveira M H. Programa de triagem neonatal para hipotireoidismo congênito no Nordeste do Brasil: critérios diagnósticos e resultados. Arq Bras de Endocrinol M etab 2008; 52(4):617-627.

43. Almeida A, Godinho TM, Teles MS, Rehem APP, Jalil HM, Fukuda TG, Araújo EP, Matos EC, Muritiba Júnior DC, Dias CPF, Pimentel HM, Fontes MIM M, Acosta AX. Avaliação do programa de triagem neonatal na Bahia no ano de 2003. Rev. Bras. Saude M ater. Infant. 2006; 6(1):85-91.
44. Ramos A, Rocha AM, Costa ADM, Benício AVL, Ramos ALC, Silva CRA. Avaliação do programa de rastreamento de doenças congênitas em Campina Grande - PB, Brasil. Arq Bras de Endocrinol Metab 2003; 47(30):280-284.

45. Ribeiro EM, Arruda AP, Alencar SC, Magalhães MTN, Montenegro AP, Almeida L, Aranha M. Neto JA. Avaliação do programa nacional de triagem neonatal no Ceará. Rev M éd de M inas Gerais 2005; 15(1-Suppl. 1):110.

46. Hegner CC, Freire R, Cintra TS, M ariani S, Goulart $S$, Lube DOF. Perfil dos pacientes portadores de hipotireoidismo congênito atendidos no programa de triagem neonatal da APAE-Vitória. Rev M éd de M inas Gerais 2005; 15(2-Suppl.1):90.

47. Carvalho MDB, Pelloso SM, Higarashi LH, Luz GS Avaliação da triagem neonatal sob a responsabilidade da equipe de enfermagem no Hospital Universitário de M aringá. Rev. M éd. de M inas Gerais 2005; 15(2-Suppl.1):50.

48. M etz M, Ranieri E, Gerace RL, Priest KR, Luke CG, Chan A. N ewborn screening in South Australia: is it universal? MJA 2003; 179(8):412-415.

49. Meirelles R, Maciel RM B, Machado-Filho A, Castro $A E$. Subsídios para regulamentação das leis que estabelecem a obrigatoriedade do diagnóstico do hipotireodismo congênito e fenilcetonúria. Arq Bras de Endocrinol M etab 1991; 35(1):12-13.

50. Sociedade Brasileira de Pediatria. [site da Internet]. [acessado 2008 ago 28]. Disponível em: http:/ /www.sbp.com.br

51. Wang W, Chen X, Zang J, He Y, Zhang P, Shen F. Development of a newborn screening laboratory quality assurance system in Shandong, China. Southeast Asian J Trop Med Publ Health 2003; 34(Suppl. 3):36-38.

Artigo apresentado em 19/11/2007

Aprovado em 30/10/2008

Versão final apresentada em 17/12/2008 